\title{
The Induction of Fos-Like Proteins in the Suprachiasmatic Nuclei and Intergeniculate Leaflet by Light Pulses in Degus (Octodon degus) and Rats
}

\author{
Kristine Krajnak, ${ }^{1}$ Lia Dickenson, and Theresa M. Lee ${ }^{2}$ \\ Department of Psychology and Reproductive Sciences Program, \\ University of Michigan, Ann Arbor, MI 48109-1109
}

\begin{abstract}
In nocturnal rodents, exposure to light results in an increase in Fos expression in two regions that receive direct retinal input: the suprachiasmatic nuclei (SCN) of the hypothalamus and the intergeniculate leaflet (IGL) of the thalamus. The induction of Fos within the SCN of nocturnal rodents is phase dependent, with light presented during the subjective night increasing Fos expression and light presented during the subjective day having little effect. By contrast, Fos expression increases in the IGL when light is presented during the subjective day or night. It is unclear whether Fos is part of the pathway mediating light-induced phase shifts in diurnal rodents. In the present study, the ability of light to induce immunostaining for Fos in the SCN and IGL was compared in diurnal rodents, Octodon degus (degus), and nocturnal rats. Degus and rats were either maintained in constant darkness or exposed to a 1-h light pulse at circadian time (CT) 4 or 16. Degus exhibit robust phase shifts at each of those circadian hours, whereas rats demonstrate phase shifts only at CT 16 . In degus, exposure to a 1-h light pulse at CT 16 resulted in an increase in the number of Fos-immunopositive ( Fos $^{+}$) cells in the ventrolateral SCN. By contrast, a 1-h light pulse at CT 4 resulted in a decrease in the number of Fos ${ }^{+}$cells in the dorsomedial portion of the SCN. In rats, a light pulse presented at CT 16 resulted in an increase in Fos ${ }^{+}$cells throughout the SCN, and a pulse at CT 4 had no effect on Fos staining. Both degus and rats showed increases in Fos expression in the IGL after light exposure at CTs 4 and 16. The authors conclude that light pulses presented at times that produce phase shifts in activity rhythms also alter Fos expression in the SCN and IGL of degus. Although these effects of light exposure on Fos expression are not identical in diurnal and nocturnal rodents, it is likely that Fos and other immediate early genes are part of the pathway mediating the effects of light in both diurnal and nocturnal rodents.
\end{abstract}

Key words Fos, immediate early genes, diurnal, degu

1. Current address: Department of Physiology, University of Kentucky, Lexington, KY 40536-0084.

2. To whom all correspondence should be addressed, at Department of Psychology and Reproductive Sciences Program, University of Michigan, 525 E. University Ave, Ann Arbor MI 48109-1109. 


\section{INTRODUCTION}

In mammals, the endogenous circadian pacemaker is located in the suprachiasmatic nuclei (SCN) of the hypothalamus. The SCN not only generate the rhythmic expression of behavioral and physiological processes but also synchronize these rhythms to the environmental light:dark (LD) cycle (Meijer and Rietveld, 1989; Miller et al., 1996). The SCN receive information about the environmental LD cycle directly from the retina via the retinohypothalamic tract (Moore and Lenn, 1972; Pickard, 1982) and indirectly via the intergeniculate leaflet (IGL) and the geniculohypothalamic tract (Card and Moore, 1982, 1988, 1989; Harrington et al., 1985, 1987). The exact mechanisms by which the SCN synchronize circadian rhythms to the LD cycle are unknown. However, numerous studies have examined the induction of immediate early genes (IEGs) in the SCN in response to light stimulation to determine whether activation of these transcription factors is part of the cascade mediating light-induced phase shifts. Although a number of IEGs, such as NGFI-A, NGFI-B, c-jun, junB, and junD, are induced by varying degrees within the $S C N$ in response to light stimulation (Rusak et al., 1992), the gene that has been studied most extensively is c-fos (for a review, see Kornhauser et al., 1993). Light pulses provided at specific times during the circadian cycle stimulate the transcription of $\mathrm{c}-$ fos and its translation to its protein product, Fos, within the SCN (Kornhauser et al., 1990; Rea, 1989; Rusak et al., 1990). Fos forms heterodimers with the protein products of the Jun family, and these heterodimers are capable of binding to the AP-1 response element to further alter gene transcription (Vogt and Bos, 1990). Although specific genes altered after Fos induction have not yet been identified in the SCN, activation of IEGs and their effects on gene transcription may be part of the pathway mediating light-induced phase shifts in circadian rhythms.

In nocturnal rodents (i.e., rats and hamsters) housed under constant dark conditions, light pulses given during the late subjective day and subjective night produce phase shifts in activity rhythms and result in an increase in Fos expression, primarily within the ventrolateral portion of the SCN (Aronin et al., 1990; Kornhauser et al., 1990; Rea, 1989, 1992; Rusak et al., 1990; Schwartz et al., 1994). Light pulses given during the subjective day (circadian times [CTs] 0-9) do not alter activity rhythms or induce Fos production (Aronin et al., 1990; Kornhauser et al., 1990;
Rusak et al., 1990). There also are a number of other studies that strongly suggest that Fos may mediate light-induced phase shifts. First, both the size of the phase shift in activity rhythms and the levels of c-fos gene expression positively correlate with the intensity of the light stimulus (Kornhauser et al., 1990). Second, at specific times during the circadian cycle, administration of the glutamate agonist $N$-methyl- $D$-aspartate mimics the effects of light on both behavioral rhythms and the induction of Fos (Ebling et al., 1991). Treatment with excitatory amino acid antagonists blocks the effects of light on both activity rhythms and Fos expression (Abe et al., 1992; Colwell et al., 1991; Rea et al., 1993). Finally, administering antisense oligonucleotides directed against $c-f o s$ and $j u n \mathrm{~B}$ to the $\mathrm{SCN}$ blocks light-induced phase shifts in activity rhythms (Wollnik et al., 1995). Thus, there appears to be a correlation between light-induced increases in Fos within the SCN and phase changes in circadian rhythms, suggesting that the induction of Fos may be part of the pathway involved in producing light-induced phase shifts in nocturnal rodents.

The SCN also receive indirect information about the LD cycle via the IGL. The IGL is a distinct region located between the dorsolateral (dLGN) and ventrolateral (vLGN) geniculate nuclei that receives direct retinal input (Hickey and Spear, 1976; Pickard, 1985). Neurons within the IGL containing neuropeptide $Y$ (Card and Moore, 1982, 1988; Harrington et al., 1985, 1987), gamma-amino butyric acid (Moore and Card, 1994; Moore and Speh, 1993), and in some species met-enkephalin (Morin et al., 1992) send projections to the ventrolateral portion of the SCN and appear to be involved in mediating photic- (Dark and Asdourian, 1975; Harrington and Rusak, 1986, 1988) and nonphotic-induced phase shifts (for a review, see Mrosovsky, 1995). Lesioning the IGL alters light-induced phase shifts (Harrington and Rusak, 1986; Johnson et al., 1989; Pickard et al., 1987) and entrainment to the environmental LD cycle (Harrington and Rusak, 1986; Johnson et al., 1989; Pickard, 1989). In nocturnal rodents, the response of the IGL to light is similar to that seen in the SCN; light exposure results in an increase in Fos expression (Edelstein and Amir, 1996; Rusak et al., 1990). However, the light-induced induction of Fos in the IGL does not appear to be phase dependent. Light pulses presented during both the subjective day and subjective night result in an increase in Fos expression in this region (Edelstein and Amir, 1996).

Although the studies mentioned heretofore demonstrate a relationship between the ability of light to 
induce Fos expression in the SCN and its ability to induce phase shifts in activity rhythms in nocturnal rodents, no experiments have been able to demonstrate this relationship in diurnal rodents. In diurnal Korean chipmunks (Eutamias asiaticus), 1-h light pulses presented during both subjective day and subjective night were capable of inducing Fos expression in the SCN. However, light pulses given at the same times did not produce consistent phase shifts in activity rhythms in these animals (Abe et al., 1995). The effects of light on the induction of Fos in the IGL were not examined in this study. Thus, based on the results of this experiment, it is difficult to determine what role, if any, Fos induction in the SCN plays in mediating light-induced phase shifts in diurnal rodents. It also is not known whether the IGL in diurnal rodents responds to light stimulation by inducing Fos expression or whether the Fos response to light is phase dependent.

Octodon degus (degus) are diurnal rodents (Goel and Lee, 1995b, 1996; Labyak and Lee, 1995) from South America with a well-characterized phase response curve to light (Lee and Labyak, 1997). Degus show phase advances in activity rhythms when exposed to light pulses between CTs 14 and 22 and show phase delays when exposed to light pulses between CTs 0 and 8 (Lee and Labyak, 1997). This is in contrast to nocturnal rodents, which exhibit no phase shifts in response to light pulses presented during the early subjective day. Because reliable phase changes in activity rhythms in response to light have been documented in degus, the effects of light on IEG induction in the SCN can be studied to determine whether cellular mechanisms responsible for producing phase shifts are similar in diurnal and nocturnal rodents. Because retinal projections to the IGL also have been described in degus (Goel and Lee, 1995a; N. Goel, T. M. Lee, and L. Smale, submitted), light-induced Fos expression also can be examined in this region. In the present experiment, we exposed rats and degus to a 1-h light pulse at CT 4 or CT 16 (times at which degus show maximal responses to light pulses) and compared the number of Fos-immunoreactive cells in the SCN and IGL of animals receiving a light pulse to that of animals that were maintained in constant darkness. If Fos or one of the Fos-related antigens (FRAs) (e.g., $F R A-1, F R A-2, F o s B$ ) is part of the pathway mediating light-induced phase shifts in degus, then light pulses presented at both times should alter the number of Fos-immunoreactive cells in the SCN and IGL of degus.

\section{METHODS}

\section{Animals}

Adult male degus ( $n=18,2-4$ years of age, mean life span $=5-7$ years) obtained from a breeding colony at the University of Michigan and adult male Sprague Dawley rats ( $n=12$, approximately 4 months of age) obtained from Charles River (Kingston, NY) were housed under a 12:12 LD cycle with lights on at 0400 $h$ and lights off (dim red illumination of less than 5 lux) at $1600 \mathrm{~h}$ for 2 weeks with food and water available ad libitum.

\section{Procedure}

After 2 weeks in the 12:12 LD cycle, animals were transferred to constant dim red illumination (DD) at the time of lights off. Animals in the experimental groups ( $n=4 /$ time/species) received a 1-h light pulse (approximately 250 lux) beginning at either CT 16 (after $28 \mathrm{~h}$ of DD exposure) or CT 4 (after $40 \mathrm{~h}$ of DD exposure). Immediately following the light pulse, animals were anesthetized with ketamine $(120 \mathrm{mg} / \mathrm{kg})$ and xylazine $(4 \mathrm{mg} / \mathrm{kg}$ ) and were perfused via the aorta with $200 \mathrm{ml}$ of $0.9 \%$ saline containing heparin $(1000 \mathrm{U} / \mathrm{L})$ followed by $250 \mathrm{ml}$ of $4 \%$ paraformaldehyde in $0.1 \mathrm{M}$ phosphate-buffered saline (PBS, $\mathrm{pH} 7.4$ ). Control animals ( $n=2$ rats $/$ time, $n=5$ degus $/$ time) remained in $\mathrm{DD}$ and were perfused at $\mathrm{CT}$ s corresponding to the times used for the experimental animals. Following perfusions, brains were postfixed in $4 \%$ paraformaldehyde for 8 to $12 \mathrm{~h}$ at room temperature and were stored at $4^{\circ} \mathrm{C}$ in $20 \%$ sucrose in $0.1 \mathrm{M}$ PBS until sectioning. Three series of coronal sections (40 $\mu \mathrm{m})$ were cut on a freezing microtome starting at the level of the striatum and continuing through the raphe. Sections were stored in cryoprotectant (Watson et al., 1986) until processed for Fos immunocytochemistry (ICC).

ICC was performed on one series of tissue sections from each animal (i.e., every third section) using standard protocols. First, sections were washed in PBS to remove any remaining cryoprotectant. All sections were then incubated in anti-Fos primary antibody diluted at 1:1000 in PBS containing 0.2\% Triton-X 100 (PBS-Tx) for $48 \mathrm{~h}$ at $4^{\circ} \mathrm{C}$. The primary antibody used was a rabbit polyclonal antibody directed against amino acid residues 3 to 16 of the human Fos protein (Santa Cruz Antibodies, Santa Cruz, CA). This anti- 

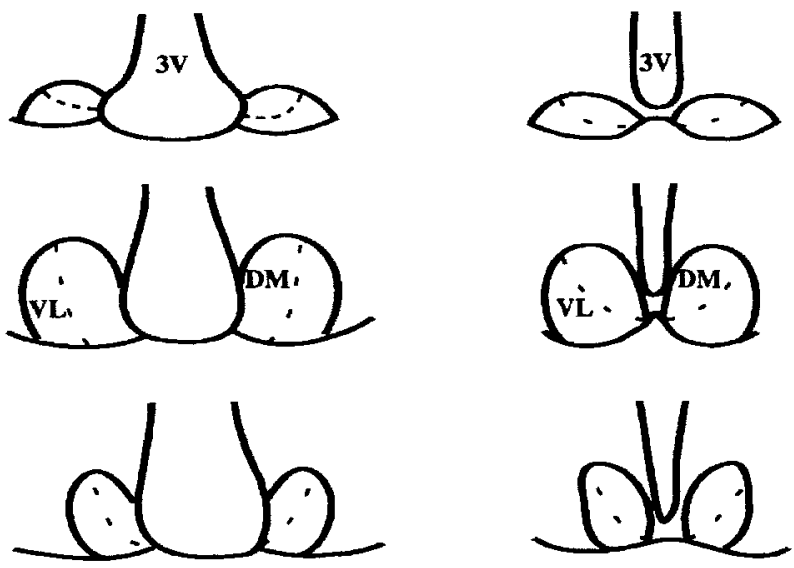

Degu

\section{Rat}

Figure 1. Representative drawings depicting the suprachiasmatic nuclei (SCN) from rostral to caudal (top to bottom) in degus and rats. The dashed lines represent the division between dorsomedial (DM) and ventrolateral (VL) SCN. These divisions were made based on the location of retinal inputs in each species (see Analysis section). $3 \mathrm{~V}=$ third ventricle.

body recognizes Fos plus other FRAs. Sections were then rinsed in PBS and incubated in anti-rabbit immuno-gamma globulin (IgG, Vectastain Elite Kit, Vector, Burlingame, CA) diluted 1:200 in PBS-Tx for $2 \mathrm{~h}$ at room temperature. This was followed by an incubation in avidin-biotin complex (1:200 in PBS-Tx, Vectastain Elite Kit) for $1.5 \mathrm{~h}$, and finally labeled cells were visualized using a peroxidase reaction with nickel chloride $(25 \mathrm{mM})$ intensified 3,3-diaminobenzadine $(0.1 \%)$ as the chromogen. All sections were mounted onto gel-coated slides and coverslipped. To determine specificity of the antibodies, sections were processed as already described, but either the primary or the secondary antibody was excluded. No labeled cells were seen in the SCN or IGL under either of these conditions.

\section{Analysis}

Sections containing the SCN were identified, and camera lucida drawings were made at a magnification of $200 \times$. Sections were designated as rostral (2 sections/animal), mid (3-4 sections/animal), or caudal (1-2 sections/animal) SCN based on the location of retinal inputs and various peptides in degus (Fig. 1) (Goel et al., submitted; Smale et al., 1993) and rats (Johnson et al., 1988; Moore, 1983). Fos staining was seen throughout the rostral-caudal extent of the SCN and in the areas lateral and dorsal to the SCN. Each section of the SCN was divided into dorsomedial and ventrolateral portions, and Fos-immunopositive $\left(\mathrm{Fos}^{+}\right)$cells were mapped and counted in each region. The ventrolateral portion of the nucleus was defined as that region receiving the majority of retinal inputs, and the dorsomedial portion was defined as that region adjacent to the ventricle that receives fewer retinal inputs in degus (Fig. 1) (Goel et al., submitted; Smale et al., 1993) and rats (Johnson et al., 1988). The number of Fos ${ }^{+}$cells in each subdivision (ventrolateral and dorsomedial) of each region (rostral, mid, and caudal) was counted by two experimenters blind to the treatment of the animals. For degus, the data from each region of the SCN were analyzed by a 2 (time) $\times$ 2 (condition) analysis of variance (ANOVA). Pairwise comparisons were made using Student's $t$ tests. Because there were only 2 control rats used per time point, and because previous work indicates that light should increase Fos ${ }^{+}$cells at CT 16 only, the number of Fos $^{+}$cells in the SCN of pulsed and control rats was analyzed using one-tailed Student's $t$ tests.

The IGL was defined as the region between the dLGN and VLGN that receives retinal inputs in both rats (Hickey and Spear, 1976; Pickard, 1985) and degus (Goel and Lee, 1995a; Goel et al., submitted; Smale et al., 1993). The number of Fos ${ }^{+}$cells was counted bilaterally at magnification of $400 \times$ by one experimenter blind to the treatment of the animals. The number of Fos ${ }^{+}$cells in the IGL of degus was analyzed using a two-way ANOVA, and the data in rats were analyzed using Student's $t$ tests as already described. Differences with $p \leq .05$ were considered significant.

\section{RESULTS}

Figure 2 shows photomicrographs taken from the mid-portion of the SCN of control $(\mathrm{A}, \mathrm{C})$ and pulsed (B,D) degus. Neither light exposure nor time had a significant effect on the number of Fos ${ }^{+}$cells in the rostral or caudal portions of the SCN. In the midportion of the SCN, the two-way ANOVA comparing the number of Fos ${ }^{+}$cells in the ventrolateral region of the SCN revealed that there was a significant interaction between time and treatment (pulsed vs. control) in degus, $F(1,14)=6.29, p<.05$. Exposure to a light pulse at CT 16 resulted in an increase in the number of Fos $^{+}$cells in the ventrolateral SCN of degus as compared to controls (Figs. 2C,D and 3A), $t(7)=2.30, p=$ 


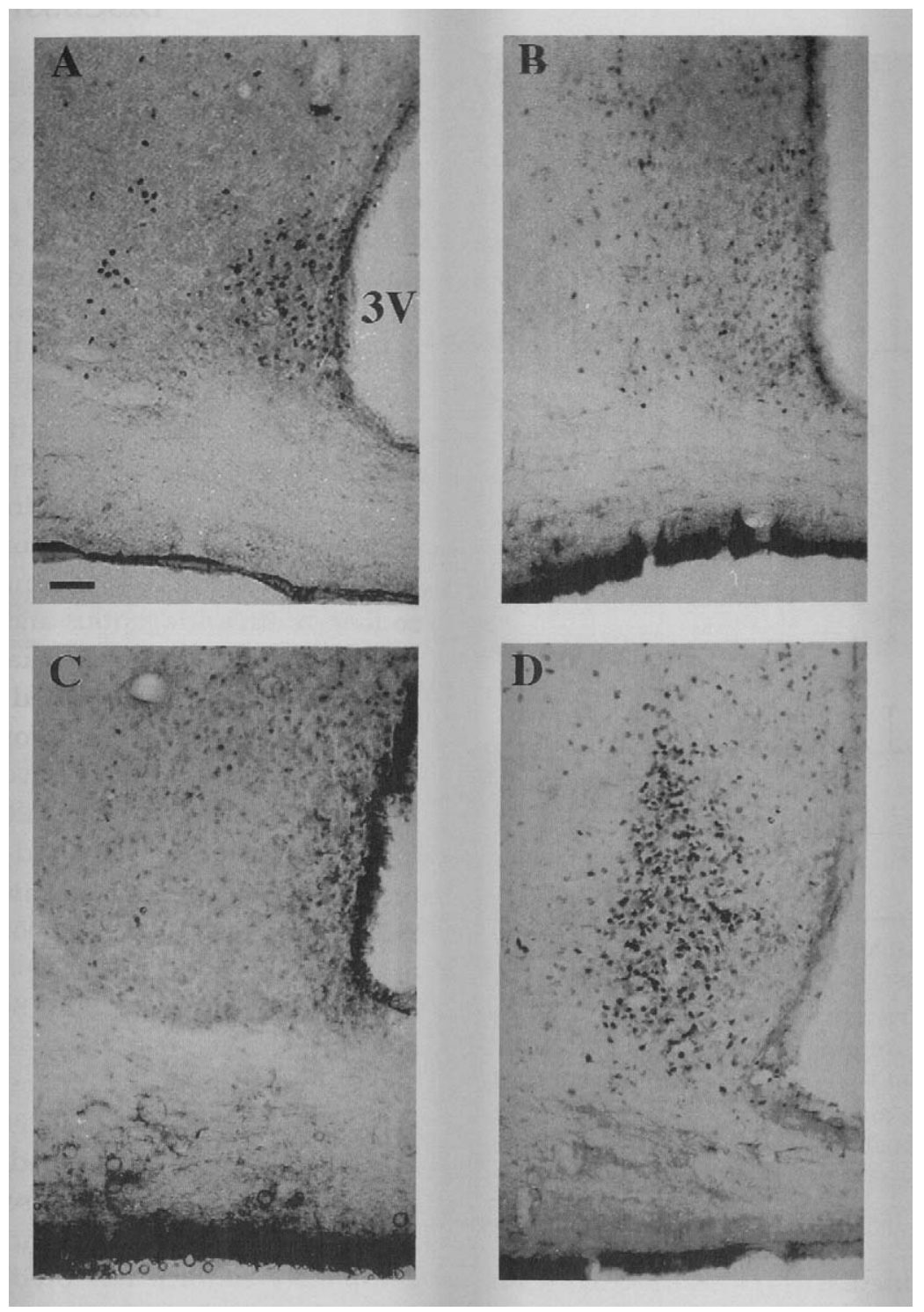

Figure 2. Photomicrographs showing Fos staining in the suprachiasmatic nuclei (SCN) of degus at circadian time (CT) 4 (A [control] and B [pulsed]) and CT 16 (C [control] and D [pulsed]). At CT 16, the majority of Fos staining was seen in the ventrolateral SCN. At CT 4, Fos staining was seen throughout the SCN in control animals, but staining was higher in the dorsomedial than in the ventrolateral portion of the nuclei. Bar $=50 \mu \mathrm{m} ; 3 \mathrm{~V}=$ third ventricle.

.05. Exposure to a light pulse at CT 4 had no effect on the number of Fos ${ }^{+}$cells in this portion of the SCN. There also was an interaction between time and treatment on the number of Fos cells in the dorsomedial portion of the SCN, $F(1,14)=4.97, p<.05$. Animals exposed to light pulses at CT 4 showed a reduction in the number of $\mathrm{Fos}^{+}$cells in the dorsomedial SCN as compared to controls (Figs. 2A,B and $3 \mathrm{~B}$ ), $t(7)=2.46, p$ $<.05$. However, pulses given at CT 16 did not alter Fos staining in this region. It also appeared as if the number of Fos $^{+}$cells in the dorsomedial SCN of CT 4 controls was greater than the number of Fos cells in the dorsomedial SCN of CT 16 controls, but this difference was not significant, $t(8)=1.91, p=.09$.

In rats as in degus, time of day or treatment did not significantly alter Fos staining in the rostral or caudal $\mathrm{SCN}$. Therefore, only the results from the mid-portion of the SCN are presented. In both the ventrolateral $(t(4)=$ 
A

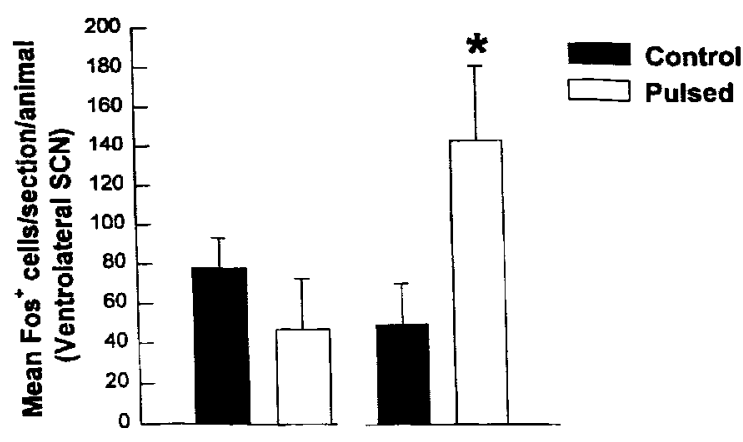

B

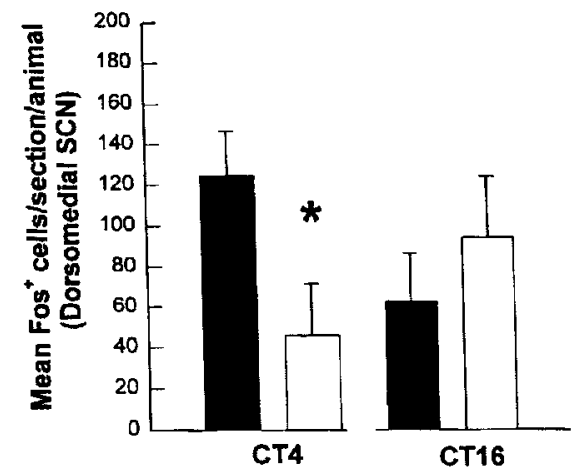

Figure 3. Fos expression in the ventrolateral $(\mathrm{A})$ and dorsomedial (B) suprachiasmatic nuclei (SCN) of control degus or degus given a 1-h light pulse at either circadian time (CT) 4 or CT 16 (means \pm SEMs of $\mathrm{Fos}^{+}$cells/section). Animals receiving a light pulse at CT 4 had a decreased number of Fos ${ }^{+}$cells in the dorsomedial SCN as compared to controls. By contrast, exposure to a light pulse at CT 16 resulted in an increase in the number of Fos ${ }^{+}$cells in the ventrolateral SCN. ${ }^{*}$ Different from same timed controls $(p \leq .05)$.

$2.37, p<.05)$ and dorsomedial $(t(4)=2.40, p<.05) \mathrm{SCN}$, pulses given at CT 16 increased the number of $\mathrm{Fos}^{+}$ cells (Figs. 4 and 5). Light pulses did not alter Fos immunostaining in either the dorsomedial or ventrolateral SCN of rats at CT 4.

Photomicrographs of Fos labeling in the IGL of rats and degus are presented in Fig. 6 . In both degus and rats, light exposure resulted in an increase in the number of $\mathrm{Fos}^{+}$cells in the IGL. In degus, the two-way ANOVA found a main effect of treatment (Fig. 7), $F(1$, $13)=4.91, p<.05$, with the number of Fos ${ }^{+}$cells being greater in the IGL of pulsed animals than control animals, but no effect of time and no interaction. In rats, there also was a significant effect of treatment on the number of Fos ${ }^{+}$cells in the IGL (Fig. 7), $t(10)=5.55$, $p<.05)$, with pulsed animals having a greater number of Fos ${ }^{+}$cells in the IGL than controls, but no effect of time of day.

\section{DISCUSSION}

In the diurnal rodent, the degu, the pattern of both endogenous and light-induced Fos expression at CT 16 is similar to that seen in nocturnal rodents (Aronin et al., 1990; Kornhauser et al., 1990; Rea, 1989, 1992; Rusak et al., 1990; Schwartz et al., 1994) and to the pattern reported in the diurnal Korean chipmunk (Abe et al., 1995). At CT 16, endogenous Fos staining is low, and exposure to a 1-h light pulse results in an increase in the number of Fos $^{+}$neurons in the ventrolateral portion of the $\mathrm{SCN}$ in degus. These results are consistent with the hypothesis that Fos induction is part of the pathway mediating light-induced phase shifts in diurnal rodents. Although induction of Fos by light exposure at CT 16 is similar in degus and other rodents, both endogenous and light-induced Fos expression at CT 4 are remarkably different from the pattern that has been reported in other rodents.

We hypothesized that if Fos was part of the pathway mediating phase shifts in degus, then light pulses presented at CT 4, which result in strong phase delays in activity rhythms (Lee and Labyak, 1997) should result in an increase in the number of Fos ${ }^{+}$cells in the $\mathrm{SCN}$. However, in degus, exposure to a 1-h light pulse at $\mathrm{CT} 4$ resulted in a decrease in Fos expression in the dorsomedial portion of the $\mathrm{SCN}$ rather than an increase (as we had expected). To our knowledge, there are no other published reports of light stimulation resulting in a decrease in IEG expression in the SCN. In fact, in all animals examined, Fos immunoreactivity is not influenced by light pulses presented during the subjective day. Therefore, although our results did not completely confirm our predictions, it is clear that light exposure does alter Fos expression in the SCN of degus at both CTs 4 and 16 and that these changes in Fos expression may be involved in mediating lightinduced phase shifts in these animals.

The mechanisms responsible for the decline in Fos staining at CT 4 are unknown. However, it is possible that exposure to light at CT 4 results in the activation of the transcriptional regulator, $\mathrm{Ca}^{2+} / \mathrm{cAMP}$ response element modulator (CREM) (Molina et al., 1993). Activation of the CAMP pathway is capable of inducing the transcription of both c-fos (Sheng and Greenberg, 1990) and a specific isoform of CREM named the inducible cAMP early repressor (ICER), which is a powerful inhibitor of cAMP-induced transcription (Molina et al., 1993; Stehle et al., 1993). Light may induce $c$-fos expression in the $\mathrm{SCN}$ by activating $\mathrm{Ca}^{2+} /$ calmodulin or cAMP pathways, which results in the phosphorylation of $\mathrm{Ca}^{2+} / \mathrm{cAMP}$ response element 


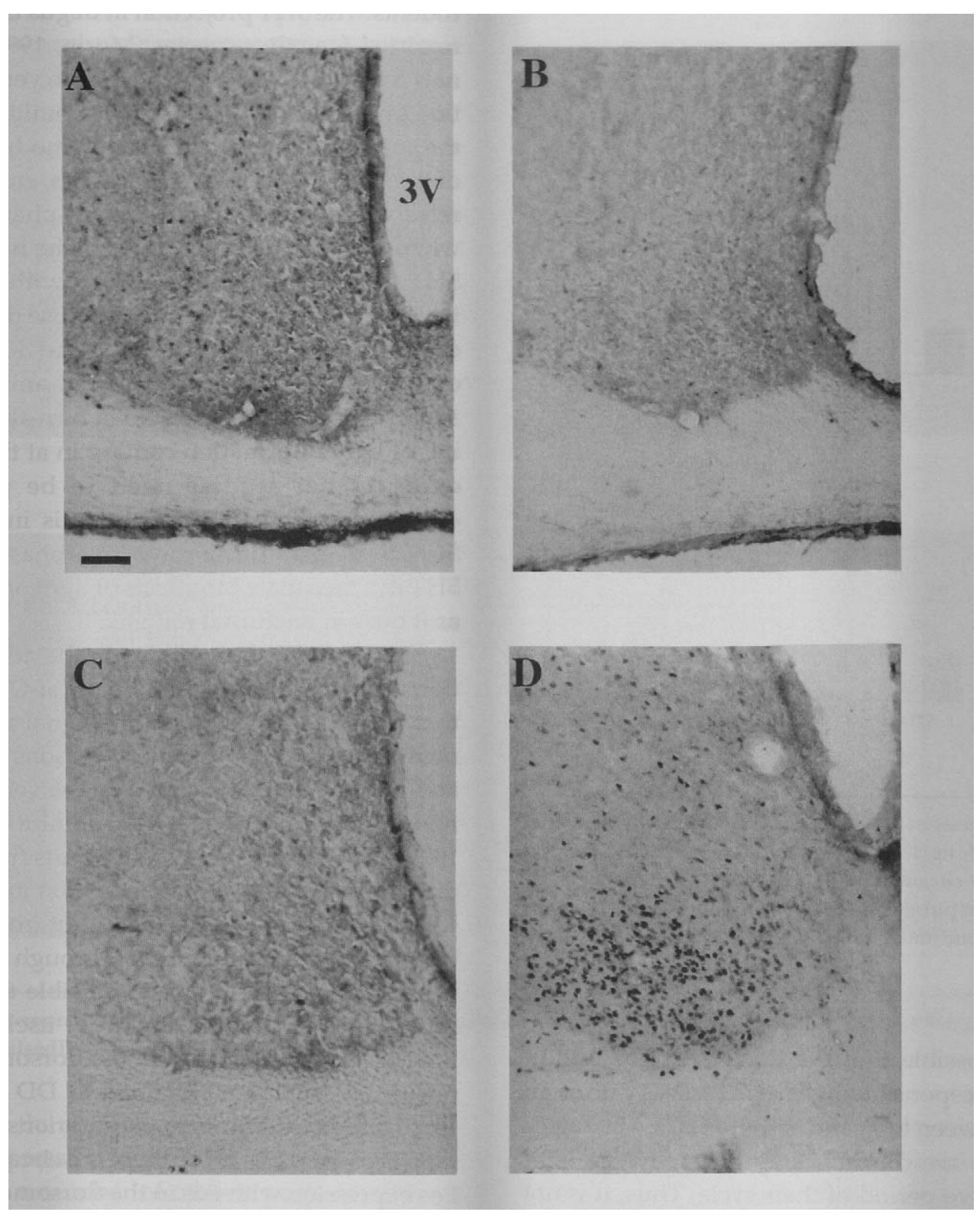

Figure 4. Photomicrographs showing Fos staining in the suprachiasmatic nuclei of rats at circadian time (CT) 4 (A [control] and B [pulsed]) and CT 16 (C [control] and D [pulsed]). Little Fos staining was seen in control animals or in animals pulsed at CT 4. However, Fos staining was seen throughout the nuclei in rats receiving a pulse at $\mathrm{CT} 16 . \mathrm{Bar}=50 \mu \mathrm{m} ; 3 \mathrm{~V}=$ third ventricle.

binding protein (CREB) (Ginty et al., 1993; Golombek and Ralph, 1994). CREB binds to the $\mathrm{Ca}^{2+} / \mathrm{cAMP}$ response element on the c-fos promoter and regulates transcription (Sheng and Greenburg, 1990). In the SCN of degus, basal Fos expression is high at CT 4, suggesting that some endogenous signal may already be activating the CAMP pathway at this time. If exposure to light results in further activation of the cAMP pathway, it is possible that ICER also is induced and that the induction of this transcriptional inhibitor pre- vents further cAMP-induced transcription of c-fos and thus Fos levels decline. Although a number of studies have shown that ICER induction and activity in the pineal gland are regulated by photoperiod (Foulkes et al., 1996; Stehle et al., 1993), experiments examining the induction and regulation of ICER by light in the SCN have not yet been performed. Thus, it remains to be determined whether this mechanism may be involved in the regulation of Fos transcription in the $\mathrm{SCN}$ of degus. 
A
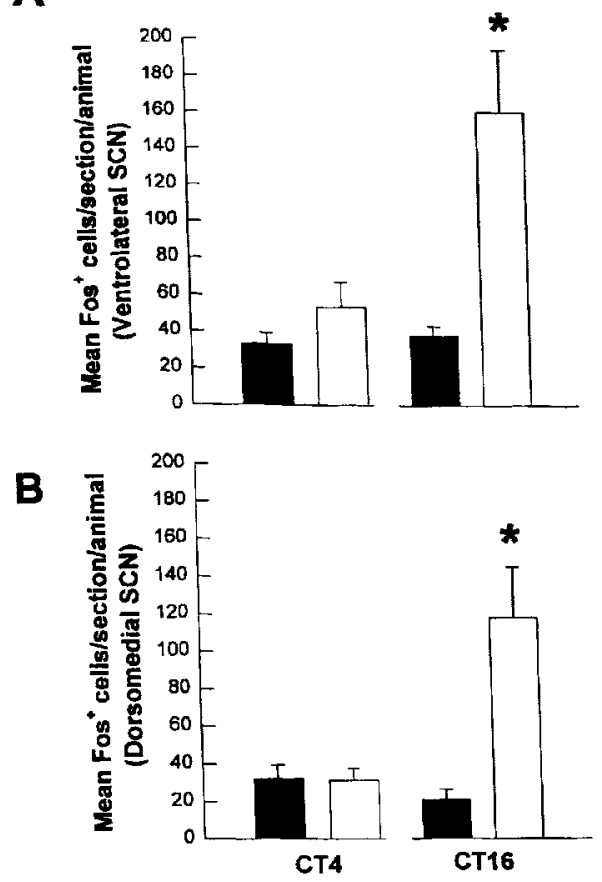

Figure 5. Fos expression in the ventrolateral (A) and dorsomedial (B) suprachiasmatic nuclei ( $\mathrm{SCN}$ ) of control rats and rats receiving a 1-h light pulse at circadian time (CT) 4 or CT 16 (means \pm SEMs of Fos ${ }^{+}$cells). Light pulses presented at CT 4 had no effect on Fos staining in either portion of the SCN. *Different from same timed controls $(p<.05)$.

It also is possible that the decline in Fos immunostaining in response to light at CT 4 was due to an interaction between light and activity at CT 4 in degus. Because degus are diurnal, they normally see light during the active period of their cycle. Thus, it is not unreasonable to think that activity may modulate the effects of light during the subjective day. One pathway by which activity may alter the effects of light on Fos staining is through the serotonergic $(5 \mathrm{HT})$ projection from the raphe (Moore et al., 1978; Morin, 1994). In hamsters, treatment with the $5 \mathrm{HT}$ precursor, tryptophan, reduces the size of light-induced phase shifts and in Fos immunostaining within the SCN (Glass et al., 1995). In in vitro slice preparations from rats, basal expression of c-fos mRNA in the dorsomedial SCN is high in untreated slices at CT 6, but treatment with the 5HT agonist quipaizine results in a decrease in Fos expression at this time (Prosser et al., 1994). Thus, it appears as if $5 \mathrm{HT}$ can inhibit both light-induced and basal expression of Fos in the SCN of nocturnal rodents. The $5 \mathrm{HT}$ projection in degus is similar to that reported for other species (Morin, 1994); 5HT terminals are found primarily within the ventrolateral portion of the nucleus (Goel et al., submitted). Therefore, the pathway by which $5 \mathrm{HT}$ could modulate the effects of light exists in degus. In hamsters, endogenous $5 \mathrm{HT}$ release is higher during the dark phase of the cycle, when animals are active. In fact, the highest levels of $5 \mathrm{HT}$ release are highly correlated with bouts of activity (Dudley and Glass, 1996). Because degus are active during the day, $5 \mathrm{HT}$ release in the SCN may be increased during the day in these animals, and this increase in 5HT release could in turn alter the processing of light information coming in at this time. However, further studies need to be performed to determine whether $5 \mathrm{HT}$ release is increased in the $\mathrm{SCN}$ of degus during the active phase and whether 5 HT can modulate the effects of light on Fos induction as it does in nocturnal rodents.

Basal levels of Fos staining in the dorsomedial portion of the SCN are high in degus at CT 4, suggesting that there is some endogenous signal activating these neurons. In degus, afferent projections from the retina and IGL terminate primarily in the ventrolateral portion of the SCN (Goel et al., submitted; Smale et al., 1993). Thus, it is unlikely that inputs from the IGL are directly responsible for the induction in Fos seen at CT 4. However, it is possible that input from the IGL may be sending an indirect signal through neurons in the ventrolateral SCN. It also is possible that a circadian signal produced within the SCN itself is generating this increase in Fos ${ }^{+}$cells in the dorsomedial SCN. In nocturnal rodents maintained in DD (Earnest et al., 1990) and in in vitro slice preparations obtained from rats (Prosser et al., 1994), there is a circadian rhythm in Fos expression with Fos in the dorsomedial portion of the nucleus being slightly higher during the day than during the night. A similar but more robust circadian rhythm in Fos expression in the SCN may be present in degus. It also is possible that the increase in immunostaining seen in the dorsomedial SCN of degus at CT 4 was not due to an increase in Fos but instead was due to the result of an increase in another FRA. For example, in rats, Fos B expression is high throughout the circadian cycle (Peters et al., 1994). To definitively determine the mechanisms responsible for the high endogenous expression of Fos immunoreactivity in the dorsomedial SCN of degus at CT 4, studies will need to be done to examine the circadian regulation of FRA expression using antibodies directed specifically against Fos versus other FRAs. 


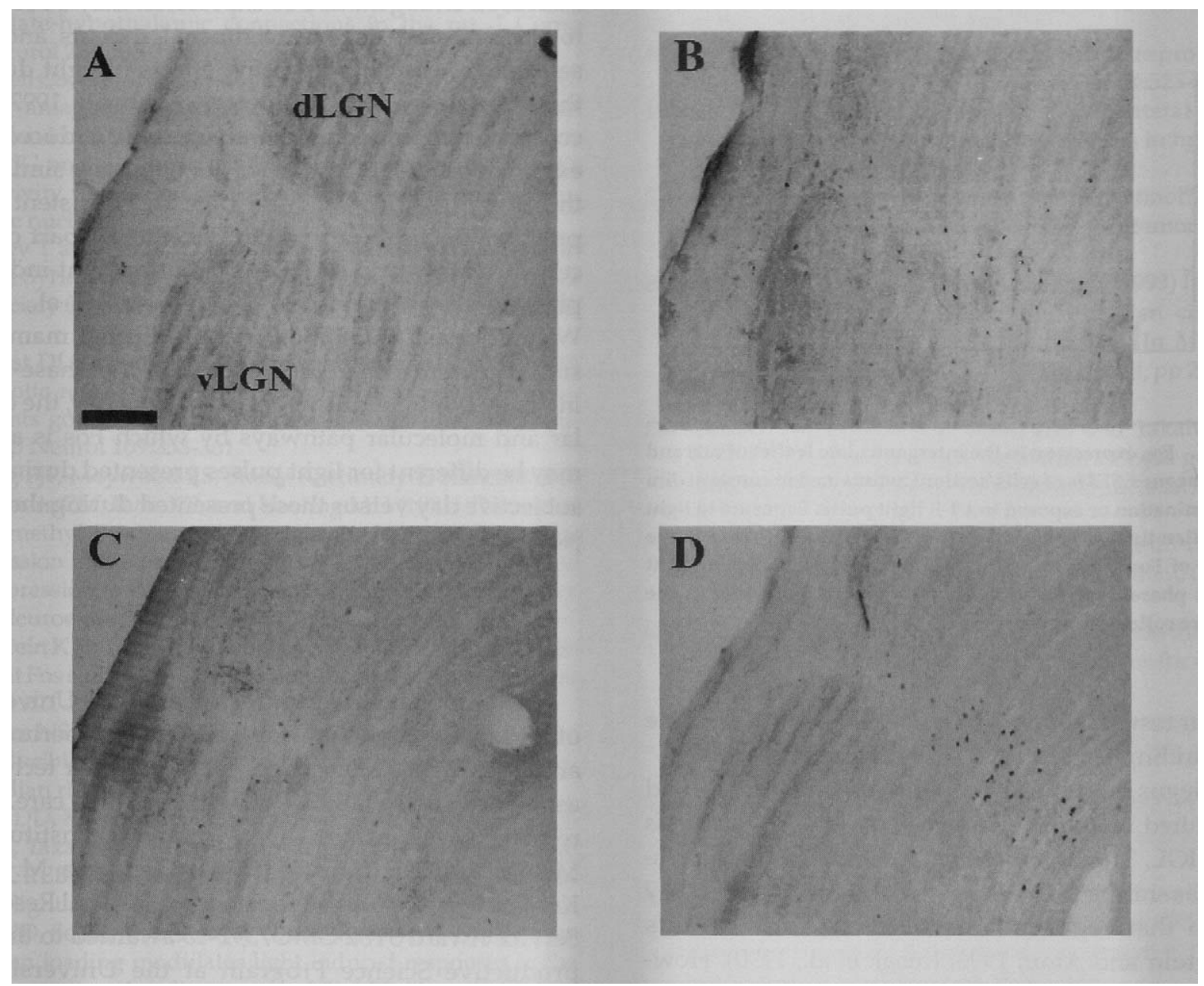

Figure 6. Photomicrographs showing Fos immunostaining in the intergeniculate leaflet of degus (A [control] and B [pulsed]) and rats (C [control] and D [pulsed]) at circadian time 16. Bar $=50 \mu \mathrm{m}$.

In this study, the patterns of endogenous and lightinduced Fos expression in the SCN and IGL of rats are consistent with a number of other reports examining Fos staining in these regions in nocturnal rodents (Aronin et al., 1990; Edelstein and Amir, 1996; Kornhauser et al., 1990; Rea, 1989, 1992; Rusak et al., 1990; Schwartz et al., 1994). In the absence of light stimulation, endogenous Fos expression in the SCN is low at both CTs 4 and 16. Only light pulses at CT 16 result in a marked increase in Fos ${ }^{+}$cells. However, in contrast to previous studies that report that increases in Fos in response to light occur primarily within the ventrolateral $\mathrm{SCN}$, in the present study light exposure increased Fos staining in both the ventrolateral and dorsomedial portions of the SCN in rats at CT 16 . Two factors may account for our findings. First, because the induction of Fos in the ventrolateral portion of the $\mathrm{SCN}$ of nocturnal rodents is so robust, and because retinal projections terminate primarily in this area of the SCN, many studies have focused on this region (Kornhauser et al., 1990; Rusak et al., 1990; Schwartz et al., 1994) and thus may not have reported the less obvious changes occurring in the dorsomedial SCN. Second, because our antibody recognizes Fos plus other FRAs, it is possible that the increase in immunostaining we saw in the dorsomedial SCN of rats was due to a light-induced increase in one of the other FRAs. In the IGL, light pulses presented at both CTs 4 and 16 resulted in an increase in Fos immunostaining in rats. Other studies also have found that the ability of light to induce Fos expression in the IGL of nocturnal rodents is not phase dependent (Edelstein and Amir, 1996; Rusak et al., 1990). Therefore, our ability to replicate many of the effects of light on Fos immunostaining in the SCN and IGL of the rat, and the fact that rats and degus were treated identically, indicates 


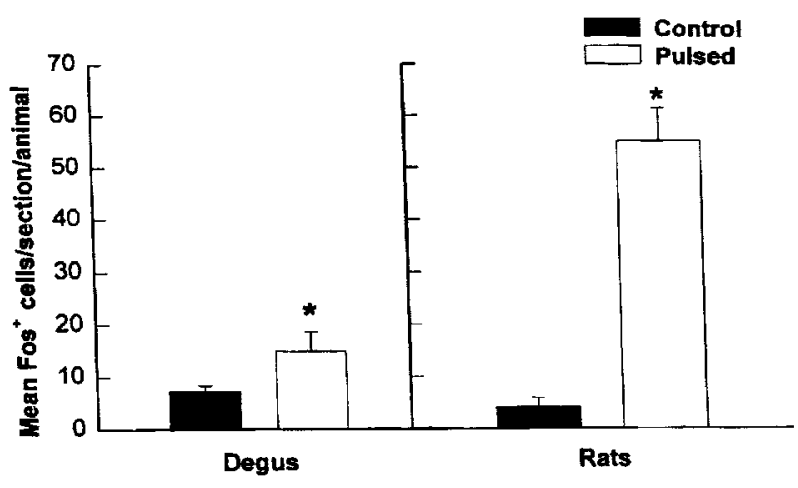

Figure 7. Fos expression in the intergeniculate leaflet of rats and degus (means \pm SEMs of cells/section) maintained in constant dim red illumination or exposed to a 1-h light pulse. Exposure to light at circadian time (CT) 4 and CT 16 resulted in an increase in the number of Fos ${ }^{+}$cells in both rats and degus. The effect of light was not phase dependent; therefore, the data presented in the figure are collapsed across time.

that our results with degus are not likely due to some confounding factor in our experimental protocol.

In degus, light pulses presented at both CTs 4 and 16 resulted in an increase in the number of Fos cells in the IGL. The light-induced increase in Fos expression was similar to that seen in rats in the current study and to that reported in other nocturnal rodents (Edelstein and Amir, 1996; Rusak et al., 1990). However, in rats, the number of Fos ${ }^{+}$cells after light exposure was approximately five times higher than that in control animals. By contrast, in degus, Fos expression only doubled after light stimulation. The blunted Fos response in the IGL of degus may be due to the fact that degus are diurnal rodents. In addition, because they normally are exposed to light throughout most of their active period, the IGL shows a blunted response to light. It also is possible that the difference in the magnitudes of the responses in rats and degus was purely a species-related difference and that a doubling of Fos expression in the degu IGL is sufficient for the processing of light information in this species.

In summary, the pattern of light-induced Fos expression in the IGL of degus is similar to that seen in rats. Thus, the role of the IGL in mediating lightinduced responses in degus and rats may be similar. In the SCN of degus, endogenous and light-induced Fos expression at CT 4 is markedly different from expression in other rodents examined previously. Based on these findings, we suggest that the differential response of the SCN to light at CT 4 and the increased endogenous Fos expression may be related to the fact that degus are diurnal rodents and are sensitive to the phase-shifting effects of light during the early subjective day (Lee and Labyak, 1997). By contrast, the patterns of basal and light-induced Fos expression at CT 16 in the SCN of degus are similar to those of other rodents. These data are consistent with previous data indicating that Fos may be part of the cellular pathway in the SCN mediating light-induced phase shifts in mammals (Kornhauser et al., 1990; Wollnik et al., 1995). However, in diurnal mammals such as degus, which are sensitive to the phase-shifting effects of light during the subjective day, the cellular and molecular pathways by which Fos is acting may be different for light pulses presented during the subjective day versus those presented during the subjective night.

\section{ACKNOWLEDGMENTS}

The authors thank Irving Zucker at the University of California, Berkeley for supplying experimental animals. Thanks also go to Kerry Grady for technical assistance and to James Donner for animal care. This research was supported by a National Institute of Mental Health grant (49089) to Theresa M. Lee. Kristine Krajnak was supported by National Research Service Award 5T32 GMO7392-15 awarded to the Reproductive Science Program at the University of Michigan.

\section{REFERENCES}

Abe H, Honma S, Shinohara K, and Honma KI (1995) Circadian modulation in photic induction of Fos-like immunoreactivity in the suprachiasmatic nucleus cells of diurnal chipmunk (Eutamius asiaticus). J Comp Physiol 176:159-167.

Abe H, Rusak B, and Robertson HA (1992) NMDA and non-NMDA receptor antagonists inhibit photic induction of Fos protein in the hamster suprachiasmatic nucleus. Brain Res Bull 28:831-835.

Aronin N, Sagar SM, Sharp FR, and Schwartz WJ (1990) Light regulates expression of a Fos-related protein in rat suprachiasmatic nuclei. Proc Natl Acad Sci USA 87:59595962.

Card JP and Moore RY (1982) Ventral lateral geniculate nucleus efferents to the rat suprachiasmatic nucleus exhibit avian pancreatic polypeptide-like immunoreactivity. J Comp Neurol 206:390-396.

Card JP and Moore RY (1988) Neuropeptide Y localization in the rat suprachiasmatic nucleus and periventricular hypothalamus. Neurosci Lett 88:241-246. 
Card JP and Moore RY (1989) Organization of lateral geniculate-hypothalamic connections in the rat. J Comp Neurol 284:135-147.

Colwell CS, Foster RG, and Menaker M(1991) NMDA receptor antagonists block the effects of light on circadian behavior in the mouse. Brain Res 554:105-110.

Dark JG and Asdourian D (1975) Entrainment of the rat's activity rhythm by cyclic light following lateral geniculate nucleus lesions. Physiol Behav 15:295-301.

Dudley T and Glass JD (1996) Endogenous 5-HT release in the Syrian hamster SCN. Paper presented at meeting of Society for Biological Rhythms, abstr 48A, Amelia Island, FL.

Earnest DJ, Iadarola M, Yeh HH, and Olschowka JA (1990) Photic regulation of c-fos expression in neural components governing the entrainment of circadian rhythms. Exp Neurol 109:353-361.

Ebling FJP, Maywood ES, Staely K, Humby T, Hancock DC, Waters CM, Evan GI, and Hasting MH (1991) The role of $N$-methyl- $D$-aspartate-type glutamatergic neurotransmission in the photic induction of immediate-early gene expression in the suprachiasmatic nuclei of the hamster. J Neuroendocrinol 3:641-652.

Edelstein K and Amir S (1996) Constant light induces persistent Fos expression in rat intergeniculate leaflet. Brain Res 731:221-225.

Foulkes NS, Duval G, and Sassone-Corsi P (1996) Adaptive inducibility of CREM as transcriptional memory of circadian rhythms. Nature 381:83-85.

Ginty DD, Kornhauser JM, Thompson MA, Bading H, Mayo KE, Takahashi JS, and Greenberg ME (1993) Regulation of CREB phosphorylation in the suprachiasmatic nucleus by light and a circadian clock. Science 260:238-241.

Glass JD, Selim M, Srkalovic G, and Rea MA (1995) Tryptophan loading modulates light-induced responses in the mammalian circadian system. J Biol Rhythms 10:80-90.

Goel N and Lee TM (1995a) Evidence of a bilateral geniculohyop thalamic tract in the diurnal rodent, Octodon degus. Paper presented at meeting of Society for Neuroscience, vol 21, abstr 185.13, San Diego.

Goel N and Lee TM (1995b) Sex differences and effects of social cues on daily rhythms following phase advances in Octodon degus. Physiol Behav 58:205-213.

Goel N and Lee TM (1996) Relationship of circadian activity and social behaviors to reentrainment rates in diurnal Octodon degus (Rodentia). Physiol Behav 59:817-826.

Golombek DA and Ralph MR (1994) KN-62, an inhibitor of $\mathrm{Ca}^{2+} /$ calmodulin kinase II, attenuates circadian responses to light. Neuroreport 5:1638-1640.

Harrington ME and Rusak B (1986) Lesions of the thalamic intergeniculate leaflet alter hamster circadian rhythms. J Biol Rhythms 1:309-325.

Harrington ME and Rusak B (1988) Ablation of the geniculohypothalamic tract alters circadian activity rhythms of hamsters housed under constant light. Physiol Behav 42:183-189.

Harrington ME, Nance DM, and Rusak B (1985) Neuropeptide $\mathrm{Y}$ immunoreactivity in the hamster geniculo-suprachiasmatic tract. Brain Res Bull 15:465-472.

Harrington ME, Nance DM, and Rusak B (1987) Doublelabeling of neuropeptide $\mathrm{Y}$ immunoreactive neurons which project from the geniculate to the suprachiasmatic nucleus. Brain Res 410:275-282.

Hickey TL and Spear PD (1976) Retinogeniculate projections in hooded and albino rats. Exp Brain Res 24:523-529.

Johnson RF, Moore RY, and Morin LP (1989) Lateral geniculate lesions alter circadian activity rhythms in hamsters. Brain Res Bull 22:411-422.

Johnson RF, Morin LP, and Moore RY (1988) Retinohypothalamic projections in the hamster and rat demonstrated using cholera toxin. Brain Res 462:301-312.

Kornhauser JM, Mayo KE, and Takahashi JS (1993) Immediate-early gene expression in a mammalian circadian pacemaker: The suprachiasmatic nucleus. In Molecular Genetics of Biological Rhythms, MW Young, ed, pp 271-307, Marcel Dekker, New York.

Kornhauser JM, Nelson DE, Mayo KE, and Takahashi JS (1990) Photic and circadian regulation of c-fos gene expression in the hamster suprachiasmatic nucleus. Neuron 5:127-134.

Labyak SE and Lee TM (1995) Estrus- and steroid-induced changes in circadian rhythms in a diurnal rodent, Octodon degus. Physiol Behav 58:573-585.

Lee TM and Labyak SE (1997) Free-running rhythms and light- and dark-pulse phase responses curves for diurnal Octodon degus. Am J Physiol 273:R278-R286.

Meijer JH and Rietveld WJ (1989) Neurophysiology of the suprachiasmatic circadian pacemaker in rodents. Physiol Rev 69:671-707.

Miller JD, Morin LP, Schwartz WJ, and Moore RY (1996) New insights into the mammalian circadian clock. Sleep 19:641-667.

Molina CA, Foulkes NS, Lalli E, and Sassone-Corsi P (1993) Inducibility and negative autoregulation of CREM: An alternative promoter directs the expression of ICER, and early response repressor. Cell 75:875-886.

Moore RY (1983) Organization and function of a central nervous system circadian oscillator: The suprachiasmatic hypothalamic nucleus. Fed Proc 42:2783-2789.

Moore RY and Card JP (1994) Intergeniculate leaflet: An anatomically and functionally distinct subdivision of the lateral geniculate complex. J Comp Neurol 344:403-430.

Moore RY, Halaris AE, and Jones BE (1978) Serotonin neurons of the midbrain raphe: Ascending projections. J Comp Neurol 146:417-438.

Moore RY and Lenn NJ (1972) A retinohypothalamic tract in the rat. J Comp Neurol 146:1-14.

Moore RY and Speh JC (1993) GABA is the principal neurotransmitter of the circadian system. Neurosci Lett 150:112-116.

Morin LP (1994) The circadian visual system. Brain Res Rev 67:102-127.

Morin LP, Blanchard J, and Moore RY (1992) Intergeniculate leaflet and suprachiasmatic nucleus organization and connections in the golden hamster. Vis Neurosci 8:219230.

Mrosovsky N (1995) A non-photic gateway to the circadian clock of hamsters. Ciba Foundation Symp 183:154-174.

Peters RV, Aronin N, and Schwartz WJ (1994) Circadian regulation of Fos B is different from c-fos in the rat suprachiasmatic nucleus. Mol Brain Res 27:243-248. 
Pickard GE (1982) The afferent connections of the suprachiasmatic nucleus of the golden hamster with emphasis on the retinohypothalamic projection. J Comp Neurol 211:65-83.

Pickard GE (1985) Bifurcating axons of retinal ganglion cells terminate in the hypothalamic suprachiasmatic nucleus and the intergeniculate leaflet of the thalamus. Neurosci Lett 55:211-217.

Pickard GE (1989) Entrainment of the circadian wheel-running activity is phase shifted by ablation of the intergeniculate leaflet. Brain Res 494:151-154.

Pickard GE, Ralph MR, and Menaker M (1987) The intergeniculate leaflet partially mediates the effects of light on circadian rhythms. J Biol Rhythms 2:35-56.

Prosser RA, Macdonald ES, and Heller HC (1994) c-fos mRNA in the suprachiasmatic nuclei in vitro shows a circadian rhythm and responds to a serotonergic agonist. Mol Brain Res 25:151-156.

Rea MA (1989) Light increases Fos-related protein immunoreactivity in the rat suprachiasmatic nuclei. Brain Res Bull 23:577-581.

Rea MA (1992) Different populations of cells in the suprachiasmatic nuclei express c-fos in association with lightinduced phase delays and advances of the free-running activity rhythm in hamsters. Brain Res 579:107-112.

Rea MA, Buckley B, and Lutton LM (1993) Local administration of EAA antagonists blocks light-induced phase shifts and c-fos expression in hamster SCN. Am J Physiol 265:R1191-R1198.

Rusak B, McNaughton L, Robertson HA, and Hunt SP (1992) Circadian variation in photic regulation of immediate- early gene mRNAs in rat suprachiasmatic nucleus cells. Mol Brain Res 14:124-130.

Rusak B, Robertson HA, Wisden W, and Hunt SP (1990) Light pulses that shift rhythms induce gene expression in the suprachiasmatic nucleus. Science 248:1237-1240.

Schwartz WJ, Takeuchi J, Shannon W, Davis EM, and Aronin $N$ (1994) Temporal regulation of light-induced Fos and Fos-like protein expression in the ventrolateral subdivision of the rat suprachiasmatic nucleus. Neuroscience 58:573-583.

Sheng M and Greenberg ME (1990) The regulation and function of c-fos and other immediate early genes in the nervous system. Neuron 4:477-485.

Smale L, Goel N, and Lee TM (1993) The suprachiasmatic nucleus and intergeniculate leaflet of the Octodon degus, a diurnal rodent. Soc Neurosci Abstr 19:1614.

Stehle JH, Foulkes NS, Molina CA, Simon-Neaux V, Pevet P, and Sassone-Corsi P (1993) Adrenergic signals direct rhythmic expression of transcriptional repressor CREM in the pineal gland. Nature 365:314-320.

Vogt PK and Bos TJ (1990) Jun: Oncogene and transcription factor. Adv Cancer Res 55:1-35.

Watson RE, Weigand SJ, Clough RW, and Hoffman GE (1986) Use of cryoprotectant to maintain long-term peptide immunoreactivity and tissue morphology. Peptides 7:155-159.

Wollnik F, Brysch W, Uhlmann E, Gillardon F, Bravo R, Zimmermann $\mathrm{M}$, Schlingensiepen $\mathrm{KH}$, and Herdegen $\mathrm{T}$ (1995) Block of $c$-fos and jun B expression by antisense oligonucleotides inhibits light-induced phase shifts of the mammalian circadian clock. Eur J Neurosci 7:388-393. 